J. Math. Soc. Japan

Vol. 36, No. 1, 1984

\title{
Basic properties of Brownian motion and a capacity on the Wiener space
}

\author{
By Masatoshi FuKUSHIMA \\ (Received Feb. 26, 1983)
}

\section{$\S 1$. Introduction.}

Among basic properties of the one-dimensional Brownian motion, we consider the property of quadratic variation, nowhere differentiability, Lévy's Hölder continuity and the law of the iterated logarithm. We shall prove that these properties hold not only almost everywhere (a.e.) with respect to the Wiener measure but also quasi everywhere (q.e.), namely, except on a polar set, with respect to the Ornstein-Uhlenbeck process on the Wiener space. We shall also consider the $d$-dimensional Brownian motion and establish q. e. statements of the unattainability of a one point set (when $d \geqq 5$ ), the transience (when $d \geqq 5$ ) and the absense of double points (when $d \geqq 7$ ).

Concerning the property of quadratic variation, D. Williams has obtained such refinement from a.e. to q.e. by a direct consideration of the OrnsteinUhlenbeck process [8] . In this paper, we instead make use of the estimates of a capacity related to the Ornstein-Uhlenbeck operator. A useful means in carrying out the computation of the estimates is a chain rule of the Dirichlet norm for composite functions. The rule has been stated already in the context of the Malliavin calculus $([\mathbf{3}],[8],[10])$ and in relation to the Dirichlet forms $([1]$, , [6] $)$.

To be precise, let us consider the $d$-dimensional Wiener space $(W, P) ; W=$ $W_{0}^{d}$ is the space of all continuous functions $w:[0, \infty) \rightarrow \boldsymbol{R}^{d}$ satisfying $w_{0}=\mathbf{0}$ and $P$ is the Wiener measure on $W . W$ is endowed with the topology of uniform convergence on every finite interval. The expectation with respect to $P$ is denoted by $E$. The $t$-th coordinate of $w \in W$ is designated by $w_{t}$ or $b_{t}(w)$ or $b(t, w) . \quad\left\{b_{t}, t \geqq 0\right\}$ performs the $d$-dimensional standard Brownian motion under the law $P$. The inner product in $L^{2}=L^{2}(W, P)$ is denoted by ( , ).

Let $L^{2}=\sum_{n=0}^{\infty} \oplus Z_{n}$ be the Wiener-Ito decomposition, $Z_{n}$ being the space of $n$ ple Wiener integrals. We consider a self-adjoint operator $\boldsymbol{A}$ on $L^{2}$ defined by

$$
A=-\sum_{n=0}^{\infty} \frac{n}{2} P_{n} \quad\left(P_{n} \text { is the projection on } Z_{n}\right) .
$$

$\boldsymbol{A}$ is non-positive definite and consequently we may consider the associated closed 
symmetric form $\mathcal{E}$ on $L^{2} ; \mathcal{E}$ is given by

$$
\left\{\begin{array}{l}
\mathscr{D}[\mathcal{E}]=\left\{u \in L^{2}: \sum_{n=0}^{\infty} n\left(P_{n} u, P_{n} u\right)<\infty\right\} \\
\mathcal{E}(u, v)=\frac{1}{2} \sum_{n=0}^{\infty} n\left(P_{n} u, P_{n} v\right) .
\end{array}\right.
$$

The domain $\mathscr{D}[\mathcal{E}]$ of $\mathcal{E}$ will be denoted by $\mathscr{F}$. As is well known, the semigroup $\left\{T_{t}=\exp (t \boldsymbol{A}), t>0\right\}$ is realized by the transition function of the Ornstein-Uhlenbeck process which is a diffusion process on $W$ (see the paragraph containing formula (1.9)). Hence $(\mathscr{F}, \mathcal{E})$ is a Dirichlet space in the sense that

$$
u \in \mathscr{F} \Longrightarrow v=(0 \vee u) \wedge 1 \in \mathscr{F}, \mathcal{E}(v, v) \leqq \mathcal{E}(u, u) .
$$

We introduce a capacity $\operatorname{Cap}(A)$ for all subsets $A$ of $W$ as follows; for an open set $A \subset W$

$$
\operatorname{Cap}(A)=\inf \left\{\mathcal{E}_{1}(u, u): u \in \mathscr{F}, u \geqq 1 P \text {-a.e. on } A\right\}
$$

and for any set $A \subset W$

$$
\operatorname{Cap}(A)=\inf \{\operatorname{Cap}(B): B \text { open, } B \supset A\},
$$

where $\mathcal{E}_{1}(u, v)=\mathcal{E}(u, v)+(u, v), u, v \in \mathscr{F}$. Cap is a non-negative increasing set function on $W$ such that

$$
\begin{aligned}
& P(A) \leqq \operatorname{Cap}(A) \quad \text { for Borel } A, \operatorname{Cap}(W)=1 \\
& A_{n} \uparrow \Longrightarrow \operatorname{Cap}\left(\bigcup_{n=1}^{\infty} A_{n}\right)=\sup _{n} \operatorname{Cap}\left(A_{n}\right) \\
& \operatorname{Cap}\left(\bigcup_{n=1}^{\infty} A_{n}\right) \leqq \sum_{n=1}^{\infty} \operatorname{Cap}\left(A_{n}\right) .
\end{aligned}
$$

Properties (1.5) and (1.6) follow from property (1.5) for open $A_{n}$ 's and the strong subadditivity of Cap for open sets, which are in turn easy consequences of the feature (1.3) of the Dirichlet form $([1 ; \S 3.1])$. A trivial but important observation we want to mention here is that the countable subadditivity (1.6) implies the capacitary version of the first Borel Cantelli lemma

$$
\sum_{n=1}^{\infty} \operatorname{Cap}\left(A_{n}\right)<\infty \Longrightarrow \operatorname{Cap}\left(\overline{\lim }_{n \rightarrow \infty} A_{n}\right)=0
$$

which will be repeatedly used in concluding our quasi-everywhere statements.

We use the term "quasi-everywhere" or "q.e." to mean "except on a subset of $W$ of capacity zero". We can now state our theorems. The first five theorems concern the one dimensional Brownian motion.

THEOREM $1\left(d=1\right.$, quadratic variation). Fix $t>0$ and consider a sequence $\Delta_{n}$ of partitions of $[0, t]: 0=t_{0}^{(n)}<t_{1}^{(n)}<\cdots<t_{m}^{(n)}=t,\left|\Delta_{n}\right|=\max _{k}\left(t_{k}^{(n)}-t_{k-1}^{(n)}\right)$. We let 


$$
S_{n}(w)=\sum_{k}\left(b\left(t_{k}^{(n)}, w\right)-b\left(t_{k-1}^{(n)}, w\right)\right)^{2}, \quad w \in W .
$$

If $\left|\Delta_{n}\right| \rightarrow 0, n \rightarrow \infty$, then $S_{n}$ converges to $t$ in capacity* and a subsequence of $S_{n}$ converges to $t$ q.e. If $\sum_{n=1}^{\infty}\left|\Delta_{n}\right|<\infty$, then $S_{n}$ converges to $t$ q.e.

THEOREM $2(d=1$, non-differentiability). $b(t)$ is nowhere differentiable in $t$ q.e.

THEOREM 3 ( $d=1$, Lévy's Hölder continuity).

$$
\lim _{\delta \downarrow 0} \sup _{\substack{0 \leq t_{1}<t_{2} \leq 1 \\ t=t_{2}-t_{1}<\delta}} \frac{\left|b\left(t_{2}\right)-b\left(t_{1}\right)\right|}{(2 t \log 1 / t)^{1 / 2}}=1 \quad \text { q.e. }
$$

THEOREM $4(d=1$, law of the iterated logarithm at 0$)$.

$$
\varlimsup_{t \downarrow 0} \frac{b(t)}{\left(2 t \log _{2} 1 / t\right)^{1 / 2}}=1 \quad \text { q.e. }
$$

THEOREM $5(d=1$, law of the iterated logarithm at $\infty)$.

$$
\varlimsup_{t \rightarrow \infty} \frac{b(t)}{\left(2 t \log _{2} t\right)^{1 / 2}}=1 \quad \text { q.e. }
$$

The next three theorems concern higher dimensional Brownian motion.

THEOREM 6 (unattainability of a one point set). Let $d \geqq 5$. Fix any point $\boldsymbol{a}=\left(a_{1}, \cdots, a_{d}\right) \in \boldsymbol{R}^{d}$ and let $\sigma_{\{\boldsymbol{a}\}}(w)=\inf \{t>0: b(t, w)=\boldsymbol{a}\} \quad$ (inf $\varnothing=\infty$ by convention). Then $\sigma_{\{a\}}=\infty$ q.e.

THEOREM 7 (Transience). Let $d \geqq 5$. Then $\lim _{t \rightarrow \infty}|b(t)|=\infty$ q.e.

THEOREM 8 (Absence of double points). Let $d \geqq 7$. Then $b(t)$ has no double point q.e.

In $\S 2$, we prove Theorem 1 and then present three propositions concerning some basic capacitary estimates. Other theorems will be proved in $\S 3$ and $\S 4$. We will refer to McKean [7], Ito-McKean [4] and Kakutani [5] for the proof of the classical a.e. statements. The idea is that our propositions of $\S 2$ together with our version (1.7) of the first Borel Cantelli lemma enable us to proceed along the same lines as in [7], [4] and [5] to our q.e. statements.

In the remainder of the introduction, we give some remarks on the probabilistic and analytic significance of the $W$-set of zero capacity. Let us consider the Ornstein-Uhlenbeck process $\left(\boldsymbol{Y}_{t}, \boldsymbol{P}\right)$ on $W$ with initial (and stationary) distribution being the Wiener measure $P$. We have then the relation

$$
\operatorname{Cap}(A)=0 \Longleftrightarrow \boldsymbol{P}\left(\boldsymbol{Y}_{t} \in A \text { for some } t>0\right)=0 .
$$

In fact, a simple application of the optional sampling theorem to a supermar-

* $\lim _{n \rightarrow \infty} \operatorname{Cap}\left(\left|S_{n}-t\right|>\varepsilon\right)=0$ for any $\varepsilon>0$. 
tingale enabies one to identify the capacitary potential of an open set $A$ with the 1-order hitting probability of $A$ with respect to the Ornstein-Uhlenbeck process ([1; Lemma 4.3.1]). The implication " $\Rightarrow$ " in $[1.8)$ is immediate from this $([\mathbf{1}$; Theorem 4.3.1]). Since our space $W$ is not locally compact, the argument of [1] does not work directly in obtaining the converse implication " $\Leftarrow$ ". However, we can show that the converse is also true by embedding $W$ continuously onto a dense Borel subset of a compact space and thus reducing the situation to the standard setting of [1] ([11]). This method of embedding has been used by Kusuoka [6] in constructing a diffusion on a Banach space.

In view of (1.8), we see that Theorem 1 includes William's result [8]. Moreover (1.8) allows us to translate each of our q.e. statements into an a.e. statement concerning the "Brownian sheet". Let $W(t, \tau), t \geqq 0, \tau \geqq 0$, be a two dimensional parameter Wiener process taking values in $\boldsymbol{R}^{d}$ defined on a probability space $(\Omega, \boldsymbol{P}): W(t, \tau)=\left(W^{1}(t, \tau), \cdots, W^{d}(t, \tau)\right)$ possesses independent components and each component $W^{i}(t, \tau)$ is continuous, vanishing on axes and centered Gaussian with $\boldsymbol{E}\left(W^{i}(t, \tau) W^{i}\left(t^{\prime}, \tau^{\prime}\right)\right)=t \wedge t^{\prime} \cdot \tau \wedge \tau^{\prime} . W(t, \tau)$ is the so called Brownian sheet. For each $t \geqq 0$ and $\omega \in \Omega, \boldsymbol{X}_{t}(\omega)=W(t, \cdot)(\omega)$ takes value in $W$ and $\left(\Omega, \boldsymbol{P}, \boldsymbol{X}_{t}\right)$ is a version of the Brownian motion on the space $W$. Following Meyer [8], we let

$$
\boldsymbol{Y}_{t}^{w}=e^{-t / 2}\left(w+\boldsymbol{X}_{e}^{t}-1\right), \quad w \in W,
$$

then $\boldsymbol{Y}_{t}^{w}$ becomes a realization of the Ornstein-Uhlenbeck process on $W$ starting at $w$ and indeed $p_{t} f(w)=\boldsymbol{E}\left(f\left(\boldsymbol{Y}_{t}^{w}\right)\right)$ is a version of $\exp (t \boldsymbol{A}) f(w)$ for the operator $\boldsymbol{A}$ of (1.1) ([8]).

Let us use a convenient fact that, as a realization of the Ornstein-Uhlenbeck process on $W$ with initial distribution being the Wiener measure $P$, we may take

$$
\boldsymbol{Y}_{t}=e^{-t / 2} W\left(e^{t}, \cdot\right), \quad t \geqq 0 .
$$

Consider a statement $S$ concerning each element $w \in W$ and let $A=\{w \in W: S(w)$ is true\}. We know from (1.8) and (1.10) that

$$
\operatorname{Cap}(A)>0
$$

if and only if

$$
\boldsymbol{P}\left(e^{-t / 2} W\left(e^{t}, \tau\right) \text { satisfies } S \text { as a function of } \tau \geqq 0 \text { for some } t>0\right)>0 .
$$

Therefore the stronger assertion

$$
\text { "S is true q.e.", namely, "Cap }\left(A^{c}\right)=0 ",
$$

holds if and only if

$$
\boldsymbol{P}\left(e^{-t / 2} W\left(e^{t}, \tau\right) \text { satisfies } S \text { as a function of } \tau \geqq 0 \text { for every } t>0\right)=1 \text {. }
$$


If $S$ holds $P$-a.e., then

$$
\boldsymbol{P}\left(e^{-t / 2} W\left(e^{t}, \tau\right) \text { satisfies } S \text { as a function of } \tau \geqq 0\right)=1 \text { for each } t>0 \text {, }
$$

because $\left\{e^{-t / 2} W\left(e^{t}, \tau\right), \tau \geqq 0\right\}$ is under $\boldsymbol{P}$ a Brownian motion for each $t>0$. However (1.13) does not always imply (1.12). For instance, let $d=1$ and take as $S$ the statement " $w_{1} \neq 0$ ". Then (1.13) is fulfilled but (1.12) is not because $\left\{e^{-t / 2} W\left(e^{t}, 1\right), t \geqq 0\right\}$ is under $\boldsymbol{P}$ a one-dimensional Ornstein-Uhlenbeck process and consequently hits the origin almost surely. In other words, the $W$-set

$$
A_{1}=\left\{w_{1}=0\right\}
$$

is $P$-negligible but of positive capacity. In the same way we can see that any finite dimensional set which is of zero Lebesgue measure and yet of positive Newtonian capacity always gives rise to a $P$-negligible $W$-set of positive capacity (see the final remark in this section).

We exhibit a more interesting $P$-negligible $W$-set of positive capacity which is not given by a finite dimensional projection as above. An intensive study of the sample function behaviours of the Brownian sheet $W(t, \tau)$ has been given by Orey-Pruitt [9]. In particular, they proved that the $\boldsymbol{P}$-measure of the $\boldsymbol{\Omega}$-set

$$
W(t, \tau)=\boldsymbol{a} \quad \text { for some }(t, \tau) \in(0, \infty) \times(0, \infty)
$$

is either 1 or 0 according as $d<4$ or $d \geqq 4$, where $\boldsymbol{a}$ is an arbitrarily fixed point of $\boldsymbol{R}^{d}$. Hence we see by (1.11) that the capacity of $W$-set

$$
A_{2}=\left\{w_{\tau}=0 \text { for some } \tau>0\right\}
$$

is either positive or zero according as $d<4$ or $d \geqq 4$, where 0 is the origin of $\boldsymbol{R}^{d}$. When $d=2$ or $3, \operatorname{Cap}\left(A_{2}\right)>0$ but, as is well known, $P\left(A_{2}\right)=0$.

Note that the statement $\operatorname{Cap}\left(A_{2}\right)=0$ for $d \geqq 5$ constitutes a part of our Theorem 6. We also note that the interpretation by (1.12) allows us to derive Theorem 5 (law of the iterated logarithm at $\infty$ ) and Theorem 7 (transience for $d \geqq 5$ ) from the corresponding statements for the Brownian sheet by Zimmerman $[12 ;(2.15)]$ and by Orey-Pruitt [9; Theorem 3.1] respectively.

Finally we add a remark that our Dirichlet form $\mathcal{E}$ of 1.2 is reduced by a finite dimensional projection to a familiar expression

$$
\mathcal{E}^{(n)}(u, v)=\frac{1}{2} \int_{R^{n}} \nabla u \cdot \nabla v d \mu \quad u, v \in C_{0}^{\infty}\left(\boldsymbol{R}^{n}\right),
$$

where $d \mu=(2 \pi)^{-n / 2} e^{-|x|^{2} / 2} d x_{1} \cdots d x_{n}$. In fact, let $\alpha_{1}, \alpha_{2}, \cdots, \alpha_{n}$ be elements of $W^{\prime}$ constituting an orthonormal system in $L^{2}([0, \infty))$ and let

$$
P_{\alpha_{1}, \cdots, \alpha_{n}}(w)=\left(\left\{\alpha_{1}, w\right\}, \cdots,\left\{\alpha_{n}, w\right\}\right) \in \boldsymbol{R}^{n}, \quad u \equiv W
$$

(see $\S 2$ for the definitions of $W^{\prime}$ and $\{$,$\} ). Then we can see by Lemma 1$ of 
$\S 2$ that

$$
\mathcal{E}\left(u \circ P_{\alpha_{1}, \ldots, \alpha_{n}}, v \circ P_{\alpha_{1}, \cdots, \alpha_{n}}\right)=\mathcal{E}^{(n)}(u, v), \quad u, v \in C_{0}^{\infty}\left(\boldsymbol{R}^{n}\right) .
$$

$\mathcal{E}^{(n)}$ is a Dirichlet form on $L^{2}\left(\boldsymbol{R}^{n} ; \mu\right)$ and indeed associated with the OrnsteinUhlenbeck process on $\boldsymbol{R}^{n}$. We can also see that the Ornstein-Uhlenbeck process on $\boldsymbol{R}^{n}$ with initial distribution $\mu$ is realized by the projection $P_{\alpha_{1}, \cdots, \alpha_{n}}\left(\boldsymbol{Y}_{t}\right)$.

The expression (1.16) tells us that a set $E \subset \boldsymbol{R}^{n}$ is of zero Newtonian capacity if and only if $E$ has zero capacity with respect to $\mathcal{E}^{(n)}$, which is in turn equivalent to the probabilistic condition that the process $P_{\alpha_{1}, \cdots, \alpha_{n}}\left(\boldsymbol{Y}_{t}\right)$ does not hit $E$ almost surely. Therefore, in view of (1.8), we can conclude that $E \subset \boldsymbol{R}^{n}$ is of zero Newtonian capacity if and only if the cylindrical $W$-set $A=P_{\alpha_{1}, \ldots, \alpha_{n}}^{-1}(E)$ satisfies $\operatorname{Cap}(A)=0$. In this sense, we may well claim that the present notion of capacity is a natural extension of the classical Newtonian capacity on the $n$ space toward the infinite dimensional space $W$.

\section{$\S 2$. Basic estimates of capacity.}

Denote by $C(W)$ the totality of (not necessarily bounded) continuous functions on $W$. Since the polynomials of the coordinate functions $b(t)=\left(b^{1}(t), b^{2}(t), \cdots, b^{d}(t)\right)$ belongs to $\mathscr{T} \cap C(W)$ and $\mathcal{E}_{1}$-dense in $\mathscr{I}$, we see in the same way as in $[1 ; \S 3.1]$ that each $u \in \mathscr{F}$ admits a quasi-continuous version $\tilde{u}$ and

$$
\operatorname{Cap}(|\tilde{u}|>\lambda) \leqq \frac{1}{\lambda^{2}} \mathcal{E}_{1}(u, u), \quad \lambda>0, \quad u \in \mathscr{F} .
$$

Theorem 1 is a straightforward application of this estimate:

Proof of Theorem 1. Suppose $d=1$. Since

$$
S_{n}-t=\sum_{k}\left[\left(b\left(t_{k}^{(n)}\right)-b\left(t_{k-1}^{(n)}\right)\right)^{2}-\left(t_{k}^{(n)}-t_{k-1}^{(n)}\right)\right] \in Z_{2},
$$

we have from the expression (1.2)

$$
\mathcal{E}_{1}\left(S_{n}-t, S_{n}-t\right)=2\left\|S_{n}-t\right\|_{L^{2}}^{2}=2 E\left(\left(S_{n}-t\right)^{2}\right) \leqq C\left|\Delta_{n}\right| \cdot t .
$$

Now (2.1) and our capacitary version of the first Borel Cantelli lemma (1.7) lead us to Theorem 1 .

q.e.d.

The proof of other theorems are not so simple and we must go a little beyond the algebraic expression (1.2) and use a chain rule in computing the Dirichlet norm. In what follows, we denote $\sqrt{\mathcal{E}(u, u)}$ and $\sqrt{\mathcal{E}_{1}(u, u)}$ by $\|u\|_{\mathcal{E}}$ and $\|u\|_{\mathcal{E}_{1}}$ respectively, $u \in \mathscr{T}$.

Following Meyer [8], we denote by $W^{\prime}$ the set of all functions

$$
\alpha:[0, \infty) \longrightarrow \boldsymbol{R}^{d}
$$

with finite variation and compact support. We define the pairing of $W^{\prime}$ and $W$ by 


$$
\{\alpha, w\}=-\int_{0}^{\infty} w_{s} \cdot d \alpha_{s}, \quad \alpha \in W^{\prime}, \quad w \in W,
$$

which is a version of the stochastic integral $\int_{0}^{\infty} \alpha_{s} \cdot d b_{s}(w)$ and belongs as a function of $w$ to the space $Z_{1} \subset \mathscr{D}(\boldsymbol{A})$. We view $W^{\prime}$ as a subspace of $L^{2}([0, \infty))=$ $L^{2}\left([0, \infty) \rightarrow \boldsymbol{R}^{d}\right)$ with inner product

$$
q(\alpha, \beta)=\int_{0}^{\infty} \alpha_{s} \cdot \beta_{s} d s .
$$

Lemma 1. Take $\alpha_{1}, \alpha_{2}, \cdots, \alpha_{n} \in W^{\prime}$ and $f \in C_{0}^{\infty}\left(\boldsymbol{R}^{n} \rightarrow \boldsymbol{R}^{1}\right)$. Then

$$
f\left(\left\{\alpha_{1}, \cdot\right\},\left\{\alpha_{2}, \cdot\right\}, \cdots,\left\{\alpha_{n}, \cdot\right\}\right) \in \mathscr{F}
$$

and

$$
\begin{aligned}
& \left\|f\left(\left\{\alpha_{1}, \cdot\right\},\left\{\alpha_{2}, \cdot\right\}, \cdots,\left\{\alpha_{n}, \cdot\right\}\right)\right\|_{\mathcal{E}}^{2} \\
& \quad=\frac{1}{2} \sum_{i, j=1}^{n} E\left[f_{x_{i}}\left(\left\{\alpha_{1}, \cdot\right\}, \cdots,\left\{\alpha_{n}, \cdot\right\}\right) f_{x_{j}}\left(\left\{\alpha_{1}, \cdot\right\}, \cdots,\left\{\alpha_{n}, \cdot\right\}\right)\right] q\left(\alpha_{i}, \alpha_{j}\right) .
\end{aligned}
$$

Proof. Consider functions $F \in \mathscr{D}(\boldsymbol{A})(\subset \mathscr{F})$ satisfying $F^{2} \in \mathscr{D}(\boldsymbol{A}), F, \boldsymbol{A} F \in$ $L^{4}(W, P)$. For such $F$ and $G$, let

$$
\langle F, G\rangle=\boldsymbol{A}(F G)-(\boldsymbol{A} F) \cdot G-F \cdot(\boldsymbol{A} G) \quad\left(\in L^{2}(W ; P)\right),
$$

then

$$
\mathcal{E}(F, G)=\frac{1}{2} E(\langle F, G\rangle)
$$

because the both hand sides equal $-E((A F) \cdot G)$. Let $F_{1}, F_{2}, \cdots, F_{n}$ be of the above type and $f$ be as in Lemma 1. Take quasi-continuous versions $\tilde{F}_{i}$ of $F_{i}$ $(\in \mathscr{F})$. Then $\tilde{F}_{i}\left(\boldsymbol{Y}_{t}\right)$ are continuous semimartingales and the Ito formula yields the chain rule $([8],[10])$ :

$$
\left\langle f\left(F_{1}, F_{2}, \cdots, F_{n}\right), G\right\rangle=\sum_{i=1}^{n} f_{x_{i}}\left(F_{1}, F_{2}, \cdots, F_{n}\right)\left\langle F_{i}, G\right\rangle .
$$

On the other hand, in view of the trivial actions of $\boldsymbol{A}$ on $Z_{1}$ and $Z_{2}$, we readily have

$$
\left\langle\left\{\alpha_{i}, \cdot\right\},\left\{\alpha_{j}, \cdot\right\}\right\rangle=q\left(\alpha_{i}, \alpha_{j}\right) .
$$

Since $F$ is closed under the composition with a uniformly Lipschitz function, the proof is complete.

q.e.d.

For an interval $I=(s, t) \subset \boldsymbol{R}$, we put

$$
X_{I}(w)=\frac{b(t, w)-b(s, w)}{\sqrt{t-s}}, \quad X_{I}(w)=\left(X_{I}^{1}(w), \cdots, X_{I}^{d}(w)\right) .
$$

Proposition 1. For disjoint intervals $I_{1}, I_{2}, \cdots, I_{N} \subset \boldsymbol{R}$ and $a_{i}^{j}<b_{i}^{j}, c_{i}^{j}>0$, $i=1,2, \cdots, N, j=1,2, \cdots, d$, we have 


$$
\begin{aligned}
\operatorname{Cap} & \left(\bigcap_{i=1}^{N} \bigcap_{j=1}^{d}\left\{a_{i}^{j}<X_{I_{i}}^{j}<b_{i}^{j}\right\}\right) \\
& \leqq\left(\frac{N d}{2 c^{2}}+1\right) P\left(\bigcap_{i=1}^{N} \bigcap_{j=1}^{d}\left\{a_{i}^{j}-c_{i}^{j}<X_{I_{i}}^{j}<b_{i}^{j}+c_{i}^{j}\right\}\right)
\end{aligned}
$$

where $c=\min _{i, j} c_{i}^{j}$.

PROOF. From Lemma 1

$$
\begin{aligned}
& \left\|f\left(X_{I_{1}}^{1}, \cdots, X_{I_{1}}^{d}, X_{I_{2}}^{1}, \cdots, X_{I_{2}}^{d}, \cdots, X_{I_{N}}^{1}, \cdots, X_{I_{N}}^{d}\right)\right\|_{\mathcal{E}}^{2} \\
& \quad=\frac{1}{2} \sum_{k=1}^{N d} E\left[f_{x_{k}}\left(X_{I_{1}}^{1}, \cdots, X_{I_{N}}^{d}\right)^{2}\right], \quad f \in C_{0}^{\infty}\left(\boldsymbol{R}^{N d} \rightarrow \boldsymbol{R}\right) .
\end{aligned}
$$

Fix $\delta>0$. For each $1 \leqq i \leqq N$ and $1 \leqq j \leqq d$, choose a $C^{\infty}$-function $f_{i}^{j}(x)$ on $\boldsymbol{R}$ such that $f_{i}^{j}(x)=1$ on $\left(a_{i}^{j}, b_{i}^{j}\right), f_{i}^{j}(x)=0$ outside $\left(a_{i}^{j}-c_{i}^{j}, b_{i}^{j}+c_{i}^{j}\right), 0 \leqq f_{i}^{j}(x) \leqq 1$ and $\left|\left(f_{i}^{j}\right)^{\prime}(x)\right|$ $\leqq \frac{1}{c-\delta}$. Then put $f\left(x_{1}, \cdots, x_{N d}\right)=f_{1}^{1}\left(x_{1}\right) \cdots f_{1}^{d}\left(x_{d}\right) f_{2}^{1}\left(x_{d+1}\right) \cdots f_{N}^{d}\left(x_{N d}\right)$. Since $F(w)=f\left(X_{I_{1}}^{1}, \cdots, X_{I_{1}}^{d}, X_{I_{2}}^{1}, \cdots, X_{I_{N}}^{d}\right) \subseteq \mathscr{I}$ is equal to 1 on the open set $B=$ $\bigcap_{i=1}^{N} \bigcap_{j=1}^{d}\left\{a_{i}^{j}<X_{I_{i}}^{j}<b_{i}^{j}\right\}$, we have, by the definition of Cap $(B)$ and (2.2),

$$
\begin{aligned}
\operatorname{Cap}(B) & \leqq \mathcal{E}_{1}(F, F)=\|F\|_{\mathcal{E}}^{2}+E\left(F^{2}\right) \\
& \leqq\left(\frac{N d}{2(c-\delta)^{2}}+1\right) P\left(\bigcap_{i=1}^{N} \bigcap_{j=1}^{d}\left\{X_{T_{i}}^{j} \in\left(a_{i}^{j}-c_{i}^{j}, b_{i}^{j}+c_{i}^{j}\right)\right\}\right) . \quad \text { q.e.d. }
\end{aligned}
$$

LEMMA 2. Let $0 \leqq s<t_{1}<t_{2}<\cdots<t_{n}$ and put $b_{s ; t_{1} \cdots t_{n}}=\left(b\left(t_{1}\right)-b(s), b\left(t_{2}\right)-b(s)\right.$, $\left.\cdots, b\left(t_{n}\right)-b(s)\right)\left(\in \boldsymbol{R}^{n d}\right)$. Suppose $f \in H_{l o c}^{1}\left(\boldsymbol{R}^{n d}\right)$ satisfies $f\left(b_{s} ; t_{1} \ldots t_{n}\right) \in L^{2}(W ; P)$ and $f_{x_{k}}\left(b_{s} ; t_{1} \cdots t_{n}\right) \in L^{2}(W ; P), 1 \leqq k \leqq n d$, then $f\left(b_{s ; t_{1} \cdots t_{n}}\right) \in \mathscr{F}$ and

$$
\begin{aligned}
& \left\|f\left(b_{s ; t_{1} \cdots t_{n}}\right)\right\|_{\mathcal{E}}^{2} \\
& =\frac{1}{2} \sum_{l, m=1}^{n} \sum_{k=1}^{d} E\left[f_{x_{(l-1) d+k}}\left(b_{s ; t_{1} \cdots t_{n}}\right) f_{x_{(m-1) d+k}}\left(b_{s ; t_{1} \cdots t_{n}}\right)\right]\left(t_{l}-s\right) \wedge\left(t_{m}-s\right) .
\end{aligned}
$$

Proof. When $f \in C_{0}^{\infty}\left(\boldsymbol{R}^{n d}\right)$, (2.3) follows from Lemma 1 by setting

$$
\alpha_{(l-1) d+k}(s)=\overbrace{\left(0, \cdots, I_{\left[s, t_{l}\right]}(s), \cdots, 0\right) .}^{k}
$$

Suppose next $f \in H^{1}\left(\boldsymbol{R}^{n d}\right)$ is of compact support. Then the sum of the right hand side of $(2.3)$ and $E\left(f\left(b_{s ; t_{1} \ldots t_{n}}\right)^{2}\right)$ is dominated by

$$
\left(\frac{1}{2} n\left(t_{n}-s\right)+1\right)\left[(2 \pi)^{n / 2}\left(t_{1}-s\right)\left(t_{2}-t_{1}\right) \cdots\left(t_{n}-t_{n-1}\right)\right]^{-d / 2}\|f\|_{H}^{2}
$$

where $\|f\|_{H^{1}}^{2}=\int_{R^{n d}}\left\{|\nabla f|^{2}+f^{2}\right\} d x$. Let $\rho_{\varepsilon}$ be a mollifier. Then $\rho_{\varepsilon} * f \in C_{0}^{\infty}\left(\boldsymbol{R}^{n d}\right)$ satisfies Lemma 2.2 and so does $f$ because $\rho_{\varepsilon} * f$ converges as $\varepsilon \downarrow 0$ to $f$ in the space $H^{1}\left(\boldsymbol{R}^{n d}\right)$. 
Now take any function $f$ satisfying the condition of the lemma and let $f^{(p)}=f \cdot g^{(p)}$ where $g^{(p)}$ is a $C^{1}$-function such that $g^{(p)}=1$ for $|x| \leqq p, g^{(p)}=0$ for $|x| \geqq p+1$ and $0 \leqq g^{(p)} \leqq 1$ and $\left|g_{x_{k}}^{(p)}\right| \leqq M$ for some $M$ independent of $x, p$ and $k$. Then $f^{(p)}$ is a function of the preceding type and

$$
E\left[\left\{\left(f^{(p)}-f^{(q)}\right)_{x_{k}}\left(b_{s} ; t_{1} \cdots t_{n}\right)\right\}^{2}\right] \leqq 8 \int_{|x| \geqq p}\left\{M^{2} f(x)^{2}+f_{x_{k}}(x)^{2}\right\} d \mu(x), \quad p \leqq q,
$$

where $\mu$ is the distribution of $b_{s ; t_{1} \cdots t_{n}}$. Hence we have Lemma 2, q.e.d.

For $b(t)=\left(b^{1}(t), \cdots, b^{d}(t)\right)$, we put

$$
M_{s, t}^{i}=\max _{s \leqq v \leqq t}\left(b^{i}(v)-b^{i}(s)\right), \quad 1 \leqq i \leqq d, \quad 0 \leqq s<t .
$$

LEMmA 3. Let $1 \leqq i \leqq d$ and $0 \leqq s<t$. If $u(x)$ is a non-negative non-decreasing convex and absolutely continuous function on $\boldsymbol{R}$ such that $u\left(M_{s, t}^{i}\right)$ and $u^{\prime}\left(M_{s, t}^{i}\right)$ are in $L^{2}(W ; P)$, then $u\left(M_{s, t}^{i}\right) \in \mathscr{F}$ and

$$
\left\|u\left(M_{s, t}^{i}\right)\right\|_{\mathcal{E}}^{2} \leqq \frac{t-s}{2} E\left(u^{\prime}\left(M_{s, t}^{i}\right)^{2}\right) .
$$

PROOF. Take $s<t_{1}<t_{2}<\cdots<t_{n} \leqq t$ and let $f\left(x_{1}, \cdots, x_{n d}\right)=u\left(x_{i} \vee x_{d+i} \vee \cdots\right.$ $\left.\vee x_{(n-1) d+i}\right)$. Then $f\left(b_{s ; t_{1} \cdots t_{n}}\right)=u\left(M_{s ; t_{1} \cdots t_{n}}^{i}\right)$ where $M_{s ; t_{1} \cdots t_{n}}^{i}=\max _{1 \leq l \leq n}\left(b^{i}\left(t_{l}\right)-b^{i}(s)\right)$. It is easy to see that $f$ satisfies the condition of Lemma 2 which implies

$$
\begin{aligned}
\left\|u\left(M_{s ; t_{1} \cdots t_{n}}^{i}\right)\right\|_{\mathcal{E}}^{2} & =\frac{1}{2} \sum_{l=1}^{n} E\left(u^{\prime}\left(M_{s ; t_{1} \cdots t_{n}}^{i}\right)^{2} ; M_{s ; t_{1} \cdots t_{n}}^{i}=b^{i}\left(t_{l}\right)-b^{i}(s)\right)\left(t_{l}-s\right) \\
& \leqq \frac{t-s}{2} E\left(u^{\prime}\left(M_{s ; t_{1} \cdots t_{n}}^{i}\right)^{2}\right) \leqq \frac{t-s}{2} E\left(u^{\prime}\left(M_{s t}^{i}\right)^{2}\right) .
\end{aligned}
$$

In the present case, the cross terms of the right hand side of (2.3) vanish because

$$
P\left(M_{s ; t_{1} \cdots t_{n}}^{i}=b^{i}\left(t_{l}\right)-b^{i}(s)=b^{i}\left(t_{m}\right)-b^{i}(s)\right) \leqq P\left(b^{i}\left(t_{l}\right)-b^{i}\left(t_{m}\right)=0\right)=0, \quad l \neq m .
$$

Let $\left\{t_{1}, t_{2}, \cdots, t_{n}\right\}$ increase to a countable dense subset of $[s, t]$. Then $u\left(M_{s ; t_{1} \cdots t_{n}}^{i}\right)$ increases to $u\left(M_{s, t}^{i}\right)$. Since $\mathcal{E}_{1}$-norm of $u\left(M_{s ; t_{1} \cdots t_{n}}^{i}\right)$ is uniformly bounded by the above estimate, the Cesaro means of a subsequence are $\mathcal{E}_{1}$-convergent to a function of $\mathscr{F}$ by virtue of the Banach-Saks theorem. The limit function must coincide with $u\left(M_{s, t}^{i}\right)$.

q.e.d.

Proposition 2. Let $1 \leqq i \leqq d$ and $0 \leqq s<t$. Then

$$
\operatorname{Cap}\left(M_{s, t}^{i}-\frac{\alpha}{2}(t-s)>\beta\right) \leqq\left(\frac{\alpha^{2}(t-s)}{4}+2\right) e^{-\alpha \beta}, \quad \alpha, \beta>0 .
$$

Proof. The right hand side equals

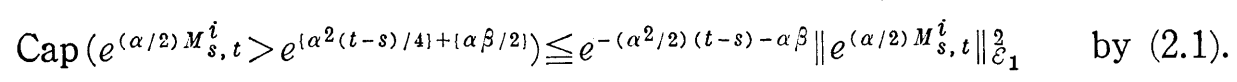

By virtue of Lemma 3, we see 


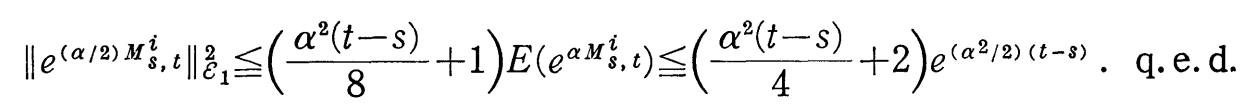

Proposition 3. For $1 \leqq i \leqq d, 0 \leqq s<t$ and $\eta>0$

$$
\begin{aligned}
& \operatorname{Cap}\left(\max _{s \leq v \leq t}\left|b^{i}(v)-b^{i}(s)\right|>\eta\right) \leqq\left(\frac{\eta^{2}}{2(t-s)}+4\right) e^{-\eta^{2} /(2(t-s))} \\
& \operatorname{Cap}\left(\max _{s \leqq v \leqq t}\left|b^{i}(t)-b^{i}(v)\right|>\eta\right) \leqq\left(\frac{\eta^{2}}{2(t-s)}+4\right) e^{-\eta^{2} /(2(t-s))} .
\end{aligned}
$$

Proof. By letting $\alpha=\frac{\eta}{t-s}$ and $\beta=\frac{\eta}{2}$ in Proposition 2, $\operatorname{Cap}\left(M_{s, t}^{i}>\eta\right) \leqq$

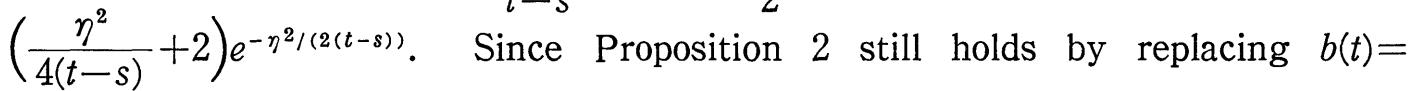
$\left(b^{1}(t), \cdots, b^{i}(t), \cdots, b^{d}(t)\right)$ with $-b(t)$, we get the first inequality. The second inequality also follows from an obvious modification of Proposition 2. q.e.d.

\section{§3. q.e. properties of the one dimensional Brownian motion.}

We assume $d=1$ and prove Theorems 2, 3, 4 and 5 stated in $\S 1$.

[3.1] Nowhere differentiability (proof of Theorem 2).

Fix a positive integer $l$ and let

$$
B_{n}=\bigcup_{1 \leqq i \leqq n-5} \bigcap_{i<j \leqq i+5}\left\{\left|b\left(\frac{j}{n}\right)-b\left(\frac{j-1}{n}\right)\right|<\frac{11 l}{n}\right\} .
$$

It suffices to prove $\operatorname{Cap}\left(\underset{n \rightarrow \infty}{\lim } B_{n}\right)=0([7 ;$ p.9]). But, by Proposition 1 , we have, for $c>0$

$$
\begin{aligned}
\operatorname{Cap}\left(B_{n}\right) & \leqq \sum_{i=1}^{n-5} \operatorname{Cap}\left(\bigcap_{i<j \leq i+5}\left\{\sqrt{n}\left|b\left(\frac{j}{n}\right)-b\left(\frac{j-1}{n}\right)\right|<\frac{11 l}{\sqrt{n}}\right\}\right) \\
& \leqq \sum_{i=1}^{n-5}\left(\frac{5 n}{2 c^{2}}+1\right) P\left(\bigcap_{i<j \leq i+5}\left\{\sqrt{n}\left|b\left(\frac{j}{n}\right)-b\left(\frac{j-1}{n}\right)\right|<\frac{11 l+c}{\sqrt{n}}\right\}\right) \\
& \leqq \frac{n\left(5 n+2 c^{2}\right)}{2 c^{2}}[P(\sqrt{n}|b(1)|<11 l+c)]^{5} \\
& =\frac{n\left(5 n+2 c^{2}\right)}{2 c^{2}}\left[\frac{1}{\sqrt{2 \pi n}} \int_{-11 l-c}^{11 l+c} e^{-\left(x^{2} / 2 n\right)} d x\right]^{5} \rightarrow 0, \quad n \rightarrow \infty
\end{aligned}
$$

q.e.d.

[3.2] Lévy's Hölder continuity (proof of Theorem 3).

Let us put

$$
F(w)=\lim _{\gamma \downarrow 0} \sup _{\substack{0 \leqq t_{1} t_{2} \leqq 1 \\ t=t_{2}-t_{1}<\gamma}} \frac{\left|b\left(t_{2}\right)-b\left(t_{1}\right)\right|}{(2 t \log 1 / t)^{1 / 2}} .
$$

PROOF OF “ $F(w) \geqq 1$ q.e.”. Let $h(t)=(2 t \log 1 / t)^{1 / 2}, 0<\delta<1$ and $0<c<\delta$, then 
by Proposition 1,

$$
\begin{aligned}
\operatorname{Cap} & \left(\max _{k 2^{n}}\left|b\left(k 2^{-n}\right)-b\left((k-1) 2^{-n}\right)\right| \leqq(1-\delta) h\left(2^{-n}\right)\right) \\
& =\operatorname{Cap}\left[\max _{k \leqq 2^{n}}\left|\frac{b\left(k 2^{-n}\right)-b\left((k-1) 2^{-n}\right)}{\sqrt{2^{-n}}}\right| \leqq(1-\delta) \sqrt{2 \log 2^{n}}\right] \\
& \leqq\left(\frac{2^{n}}{4 c^{2} \log 2^{n}}+1\right) P\left[\max _{k \leqq 2^{n}}\left|\frac{b\left(k 2^{-n}\right)-b\left((k-1) 2^{-n}\right)}{\sqrt{2^{-n}}}\right| \leqq(1-\delta+c) \sqrt{2 \log 2^{n}}\right] \\
& \leqq\left(\frac{2^{n}}{4 c^{2} n \log 2}+1\right) \exp \left(-2^{n(\delta-c)}\right),
\end{aligned}
$$

the last inequality holding for sufficiently large $n$ according to $[7 ; \mathrm{p} .15]$. Since the sum in $n$ of the last expression is finite, we have from (1.7)

$$
\max _{k \leq 2^{n}} \frac{\left|b\left(k 2^{-n}\right)-b\left((k-1) 2^{-n}\right)\right|}{h\left(2^{-n}\right)}>1-\delta \quad \text { from some } n \text { on, q.e., }
$$

which implies the desired inequality because $\delta>0$ is arbitrary.

q. e.d.

Proof of " $F(w) \leqq 1$ q.e.". By Proposition 3, we have for $\varepsilon>0$ and $0 \leqq s<t$

$$
\operatorname{Cap}[|b(t)-b(s)|>(1+\varepsilon) h(t-s)] \leqq\left((1+\varepsilon)^{2} \log \frac{1}{t-s}+4\right)(t-s)^{(1+\varepsilon)^{2}} .
$$

Hence, using the subadditivity of the capacity, we find

$$
\begin{aligned}
\text { Cap } & {\left[\max _{\substack{0<k=j-i \leq 2 n \delta \\
0<i<j \leq 2 n}} \frac{\left|b\left(j 2^{-n}\right)-b\left(i 2^{-n}\right)\right|}{h\left(k 2^{-n}\right)} \geqq 1+\varepsilon\right] } \\
& \leqq \sum_{\substack{0<k=j-i \leq 2 \leq n \delta \\
0<i<j \leq 2 n}} \operatorname{Cap}\left(\frac{\left|b\left(j 2^{-n}\right)-b\left(i 2^{-n}\right)\right|}{h\left(k 2^{-n}\right)} \geqq 1+\varepsilon\right) \\
& \leqq 2^{n} \sum_{1 \leq k \leq 2}\left\{(1+\varepsilon)^{2} \log \frac{2^{n}}{k}+4\right\}\left(k 2^{-n}\right)^{(1+\varepsilon)^{2}} \\
& \leqq 2^{n(1+\delta)}\left\{(1+\varepsilon)^{2} n \log 2+4\right\} 2^{-n(1-\delta)(1+\varepsilon)^{2}} .
\end{aligned}
$$

Choose small $\delta>0$ satisfying $(1+\delta)<(1-\delta)(1+\varepsilon)^{2}$, then by $(1.7)$ again, we see that, for q. e. $w \in W$, there exists $m=m(w)$ such that for any $n \geqq m(w)$

$$
\left|b\left(j 2^{-n}\right)-b\left(i 2^{-n}\right)\right|<(1+\varepsilon) h\left(k 2^{-n}\right), 0<i<j \leqq 2^{n}, k=j-i \leqq 2^{n \grave{\delta}} .
$$

But we have for such $w F(w) \leqq 1+3 \varepsilon+2 \varepsilon^{2}$ ([7; p. 16]).

q.e.d.

[3.3] Law of the iterated logarithm at 0 (proof of Theorem 4).

Let us put

$$
F(w)=\varlimsup_{t \downarrow 0} \frac{b(t)}{\left(2 t \log _{2} 1 / t\right)^{1 / 2}} .
$$

ProOF OF “ $F(w) \leqq 1$ q.e.". Let $h(t)=\left(2 t \log _{2} 1 / t\right)^{1 / 2}$ and take just as in 
$\left[7 ;\right.$ p. 13] $0<\theta<1,0<\delta<1, \alpha=(1+\delta) \theta^{-n} h\left(\theta^{n}\right), \beta=h\left(\theta^{n}\right) / 2, t=\theta^{n-1}$ and $s=0$ so that

$$
\left(\frac{\alpha^{2}(t-s)}{4}+2\right) e^{-\alpha \beta}=C_{1}\left(\log n+C_{2}\right) n^{-(1+\tilde{o})}
$$

is a general term of a convergent sum. By Proposition 2 and (1.7),

$$
\max _{0<v<\theta n-1} b(v) \leqq\left[\frac{1+\delta}{2 \theta}+\frac{1}{2}\right] h\left(\theta^{n}\right) \quad \text { from some } n \text { on, q.e. }
$$

The rest of the proof is the same as in [7].

PROOF OF " $F(w) \geqq 1$ q.e.". We choose $0<\theta<1$ and put $\xi=\log 1 / \theta, h(t)=$ $\left(2 t \log _{2} 1 / t\right)^{1 / 2}$ and $W_{k}=\left\{b\left(\theta^{k}\right)-b\left(\theta^{k+1}\right) \leqq(1-\sqrt{\theta}) h\left(\theta^{k}\right)\right\}$. By Proposition 1

$$
\begin{aligned}
\operatorname{Cap} & \left(\bigcap_{k=l}^{n} W_{k}\right)=\operatorname{Cap}\left[\bigcap_{k=l}^{n} \frac{b\left(\theta^{k}\right)-b\left(\theta^{k+1}\right)}{\sqrt{\theta^{k}(1-\theta)} \leqq \frac{1-\sqrt{ } \theta}{\sqrt{ } 1-\theta}}(2 \log \xi k)^{1 / 2}\right] \\
& \leqq\left(\frac{(1-\theta) n}{4 c^{2} \log \xi l}+1\right) P\left[\bigcap_{k=l}^{n}\left\{\frac{b\left(\theta^{k}\right)-b\left(\theta^{k+1}\right)}{\sqrt{\theta^{k}(1-\theta)}}<\frac{1-\sqrt{\theta}+c}{\sqrt{ } 1-\theta}(2 \log \xi k)^{1 / 2}\right\}\right] \\
& =\left(\frac{(1-\theta) n}{4 c^{2} \log \xi l}+1\right) \prod_{k=l}^{n}\left(1-I_{k}\right) \leqq\left(\frac{(1-\theta) n}{4 c^{2} \log \xi l}+1\right) \exp \left(-\sum_{k=l}^{n} I_{k}\right) .
\end{aligned}
$$

Here $I_{k}$ denotes the integral of $\frac{\exp \left(-c^{2} / 2\right)}{\sqrt{2 \pi}} d c$ on $\left[\frac{1-\sqrt{\theta}+c}{\sqrt{1-\theta}} \sqrt{2} \log \xi k, \infty\right)$. Choose $c>0$ so small that $\frac{1-\sqrt{\theta}+c}{\sqrt{1-\theta}}<1$, then

$$
\sum_{k=l}^{n} I_{k} \geqq C \sum_{k=l}^{n} \frac{k^{-(1-\sqrt{\theta}+c)^{2} /(1-\theta)}}{\sqrt{\log k}} \geqq C^{\prime}\left(n^{1-\kappa}-l^{1-\kappa}\right),
$$

where $\kappa$ is such that $\frac{(1-\sqrt{\theta}+c)^{2}}{\sqrt{1-\theta}}<\kappa<1$. Hence

$$
\operatorname{Cap}\left(\bigcap_{k=l}^{\infty} W_{k}\right) \leqq \operatorname{Cap}\left(\bigcap_{k=l}^{n} W_{k}\right) \leqq C^{\prime} n e^{-C^{\prime}\left(n^{1-k}-l^{1-k}\right)} \rightarrow 0, \quad n \rightarrow \infty .
$$

Therefore $\operatorname{Cap}\left(\lim _{k \rightarrow \infty} W_{k}\right)=0$, namely, $b\left(\theta^{n}\right)-b\left(\theta^{n+1}\right)>(1-\sqrt{ } \theta) h\left(\theta^{n}\right)$ infinitely often q.e. The rest of the proof is again the same as in $[7 ;$ p.14]. q.e.d.

[3.4] Law of the iterated logarithm at $\infty$ (proof of Theorem 5).

Trivial modifications of the proof of Theorem 4 suffice. In fact, we let $h(t)=\left(2 t \log _{2} t\right)^{1 / 2}, \theta>1,0<\delta<1, \alpha=(1+\delta) \theta^{-n} h\left(\theta^{n}\right), \quad \beta=h\left(\theta^{n}\right) / 2, t=\theta^{n+1}$ and $s=0$

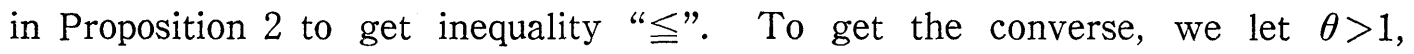
$\xi=\log \theta$ and $W_{k}=\left\{b\left(\theta^{k+1}\right)-b\left(\theta^{k}\right) \leqq(1-1 / \sqrt{\theta}) h\left(\theta^{k+1}\right)\right\}$ q.e.d.

REMARK. Here is another way to prove Theorem 5, Note that we could start with, instead of $W=W_{0}^{d}$, the space $\widetilde{W}=C\left((0, \infty) \rightarrow \boldsymbol{R}^{d}\right)$ and the Wiener 
measure $P$ on $\widetilde{W}$. Then $T w(t)=t w(1 / t), t>0$, defines a $P$-measure preserving transform $T$ on $\widetilde{W}$ which also preserves the associated capacity $\widetilde{\text { Cap }}$ on $\widetilde{W}$, because the induced transform on $L^{2}(\widetilde{W}, P)$ makes spaces $Z_{n}$ and consequently the Dirichlet form $(1.2)$ invariant. Hence Theorem 5 (for $\widetilde{W}$ ) follows from Theorem 4 (for $\widetilde{W}$ ) directly. Now our space $W$ is continuously embedded into $\widetilde{W}$ and $P(\widetilde{W}-W)=0$. This means that $\operatorname{Cap}(B) \leqq \widetilde{C a p}(B)$ for $B \subset W$ (actually the equality holds by virtue of the compactification argument $([11])$ and we can recover q.e. statements on $W$ from those on $\widetilde{W}$. It may be also possible to derive the second inequality of Proposition 3 from the first one in a similar way.

\section{$\S 4$. q.e. properties of higher dimensional Brownian motions.}

As in $\S 2$, we write as $b(t)=\left(b^{1}(t), \cdots, b^{d}(t)\right)$. We first note an obvious remark that each component $b^{i}(t), 1 \leqq i \leqq d$, satisfies the q.e. statements of Theorems 1 through 5. We shall now prove Theorems 6,7 and 8 stated in $\S 1$.

[4.1] Unattainability of a one point set (proof of Theorem 6).

Assume $d \geqq 5$. Fix $\boldsymbol{a}=\left(a_{1}, \cdots, a_{d}\right) \in \boldsymbol{R}^{d}$ and define as in $[4 ;$ p. 62]

$$
B_{n}=\bigcup_{n \leqq k<2 n} \bigcap_{1 \leqq i \leqq d}\left\{\left|b^{i}\left(\frac{k}{n}\right)-a_{i}\right|<\sqrt{\frac{3 \log n}{n}}\right\} .
$$

It suffices to show $\lim _{n \rightarrow \infty} \operatorname{Cap}\left(B_{n}\right)=0$, because, by virtue of Theorem 3 holding for each component $b^{i}(t)$, we see that the set

$$
\left\{\left(b^{1}(t), \cdots, b^{d}(t)\right)=\left(a_{1}, \cdots, a_{d}\right) \text { for some } t \in[1,2)\right\}
$$

is contained q.e. in the set $\lim _{n \rightarrow \infty} B_{n}$.

By Proposition 1, we have for each $k$,

$$
\begin{aligned}
& \operatorname{Cap}\left(\bigcap_{i \leqslant d}\left\{\left|b^{i}\left(\frac{k}{n}\right)-a_{i}\right|<\sqrt{\frac{3 \log n}{n}}\right\}\right) \\
& =\operatorname{Cap}\left[\bigcap_{i \leq d}\left\{\sqrt{\frac{n}{k}} b^{i}\left(\frac{k}{n}\right) \in\left(\sqrt{\frac{n}{k}} a_{i}-\sqrt{\frac{3 \log n}{k}}, \sqrt{\frac{n}{k}} a_{i}+\sqrt{\frac{3 \log n}{k}}\right)\right\}\right] \\
& \leqq\left(\frac{k d}{2 c^{2} \frac{\log n}{10}}+1\right) P\left[\bigcap _ { i \leq d } \left\{\sqrt { \frac { n } { k } } b ^ { i } ( \frac { k } { n } ) \in \left(\sqrt{\frac{n}{k}} a_{i}-(\sqrt{3}+c) \sqrt{\frac{\log n}{k}},\right.\right.\right. \\
& \left.\left.\left.\sqrt{\frac{n}{k}} a_{i}+(\sqrt{3}+c) \sqrt{\frac{\log n}{k}}\right)\right\}\right] \\
& \leqq 2\left(\frac{k d}{2 c^{2} \log n}+1\right)(\sqrt{3}+c)\left(\frac{\log n}{2 \pi k}\right)^{d / 2} .
\end{aligned}
$$


Hence $\operatorname{Cap}\left(B_{n}\right) \leqq C n\left(\frac{\log n}{n}\right)^{(d / 2)-1} \rightarrow 0, \quad n \rightarrow \infty$.

[4.2] Transience (proof of Theorem 7).

Assume $d \geqq 5$. If we let, just as in [5], $t_{k}=k^{3 / 4}, k=1,2, \cdots$, and $\gamma_{k}=$ $\operatorname{Cap}\left(\left|b^{i}(t)\right|<M, 1 \leqq i \leqq d\right.$, for some $\left.t \in\left[t_{k}, t_{k+1}\right]\right)$ for $M>0$, then

$$
\begin{aligned}
\gamma_{k} \leqq & \operatorname{Cap}\left(\left|b^{i}\left(t_{k}\right)\right|<2 M, 1 \leqq i \leqq d\right) \\
& +\sum_{i=1}^{d} \operatorname{Cap}\left(\max _{t_{k} \leqq t \leqq t_{k+1}}\left|b^{i}(t)-b^{i}\left(t_{k}\right)\right|>M\right) .
\end{aligned}
$$

The first term of the right hand side of (4.1) equals

$$
\begin{aligned}
\operatorname{Cap} & \left(\frac{1}{\sqrt{t_{k}}}\left|b^{i}\left(t_{k}\right)\right|<\frac{2 M}{\sqrt{t_{k}}}, 1 \leqq i \leqq d\right) \\
\quad \leqq & \left(\frac{d t_{k}}{2 M^{2}}+1\right) P\left(\frac{1}{\sqrt{t_{k}}}\left|b^{i}\left(t_{k}\right)\right|<\frac{3 M}{\sqrt{t_{k}}}, 1 \leqq i \leqq d\right) \\
\quad \leqq & \left.\frac{d t_{k}}{2 M^{2}}+1\right)\left(\frac{6 M}{\sqrt{2 \pi t_{k}}}\right)^{d} \leqq C_{1} k^{-3(d-2) / 8}
\end{aligned}
$$

by virtue of Proposition 1. By Proposition 3, the second term of (4.1) is dominated by

$$
d\left(\frac{M^{2}}{2\left(t_{k+1}-t_{k}\right)}+4\right) \exp \left(-\frac{M^{2}}{2\left(t_{k+1}-t_{k}\right)}\right) \leqq C_{2} k^{1 / 4} \exp \left(-C_{3} k^{1 / 4}\right) .
$$

Hence $\sum_{k=1}^{\infty} \gamma_{k}<\infty$ and we see from (1.7) that, for q.e. $w \in W$, there exists $k_{0}=$ $k_{0}(w)$ and $\sqrt{\sum_{i=1}^{d} b^{i}(t)^{2}}>M$ for any $t>t_{k_{0}}$.

q.e.d.

[4.3] Absence of double points (proof of Theorem 8).

Assume $d \geqq 7$. We still follow [5] to consider disjoint intervals $I=\left(s_{0}, s_{1}\right)$ and $J=\left(t_{0}, t_{1}\right)$ with $s_{1}<t_{0}$ and let $\gamma=\operatorname{Cap}\left(b^{i}(s)=b^{i}(t), 1 \leqq i \leqq d\right.$, for some $s \in I$ and $t \in J)$. Then, for $\eta>0$,

$$
\begin{aligned}
\gamma \leqq & \operatorname{Cap}\left(\left|b^{i}\left(s_{1}\right)-b^{i}\left(t_{0}\right)\right|<2 \eta, \quad i=1,2, \cdots, d\right) \\
& +\sum_{i=1}^{d} \operatorname{Cap}\left(\max _{s \in I}\left|b^{i}\left(s_{1}\right)-b^{i}(s)\right|>\eta\right)+\sum_{i=1}^{d} \operatorname{Cap}\left(\max _{t \in J}\left|b^{i}(t)-b^{i}\left(t_{0}\right)\right|>\eta\right) \\
\leqq & \frac{d\left(t_{0}-s_{1}\right)+2 \eta^{2}}{2 \eta^{2}}\left(\frac{6 \eta}{\sqrt{ } 2 \pi\left(t_{0}-s_{1}\right)}\right)^{d} \\
& +d\left(\frac{\eta^{2}}{2\left(s_{1}-s_{0}\right)}+4\right) \exp \left(-\frac{\eta^{2}}{2\left(s_{1}-s_{0}\right)}\right) \\
& +d\left(\frac{\eta^{2}}{2\left(t_{1}-t_{0}\right)}+4\right) \exp \left(-\frac{\eta^{2}}{2\left(t_{1}-t_{0}\right)}\right),
\end{aligned}
$$


where we have used Proposition 1, the second inequality of Proposition 3 and the first one of Proposition 3 successively.

Divide $I$ and $J$ into $p$-subintervals of equal length:

$$
I=\sum_{k=1}^{p} I_{k}, \quad J=\sum_{k=1}^{p} J_{k}, \quad\left|I_{k}\right|=\frac{|I|}{p}, \quad\left|J_{k}\right|=\frac{|J|}{p}, \quad k=1,2, \cdots, p .
$$

Applying the above inequality to $I_{k}$ and $J_{l}$, we get

$$
\begin{aligned}
\gamma \leqq \sum_{k=1}^{p} & \sum_{l=1}^{p} \operatorname{Cap}\left(b^{i}(s)=b^{i}(t), 1 \leqq i \leqq d, \text { for some } s \in I_{k} \text { and } t \in J_{l}\right) \\
\leqq p^{2}\{ & \frac{6^{d}\left(d\left(t_{1}-s_{0}\right)+2 \eta^{2}\right)}{2\left(2 \pi\left(t_{0}-s_{1}\right)\right)^{d / 2}} \eta^{d-2}+d\left(\frac{p \eta^{2}}{2\left(s_{1}-s_{0}\right)}+4\right) \\
& \left.\times \exp \left(-\frac{p \eta^{2}}{2\left(s_{1}-s_{0}\right)}\right)+d\left(\frac{p \eta^{2}}{2\left(t_{1}-t_{0}\right)}+4\right) \exp \left(-\frac{p \eta^{2}}{2\left(t_{1}-t_{0}\right)}\right)\right\}
\end{aligned}
$$

which tends to zero if we set $\eta=p^{-\sigma}$ with $\frac{2}{d-2}<\sigma<\frac{1}{2}$ and let $p \rightarrow \infty$.

q.e.d.

\section{References}

[1] M. Fukushima, Dirichlet forms and Markov processes, North Holland and Kodansha, 1980.

[2] M. Fukushima, Capacitary maximal inequalities and an ergodic theorem, Proceedings of the 4-th USSR-Japan Symposium on Probability Theory and Mathematical Statistics, Lecture Notes in Math., 1021, Springer, 1983.

[3] N. Ikeda and S. Watanabe, Stochastic differential equations and diffusion processes, North Holland and Kodansha, 1981.

[4] K. Ito and H. P. McKean, Diffusion processes and their sample paths, Springer, 1965.

[5] S. Kakutani, On Brownian motion in $n$-space, Proc. Acad. Japan, 20 (1944), 648-652.

[6] S. Kusuoka, Dirichlet forms and diffusion processes on Banach spaces, J. Fac. Sci. Univ. Tokyo Sect. IA, 29 (1982), 79-95.

[7] H. P. McKean, Stochastic integrals, Academic Press, 1969.

[8] P. A. Meyer, Note sur les processus d'Ornstein-Uhlenbeck (Appendice: Un resultat de D. Williams), Séminaire de Probabilités XVI 1980/81, Lecture Notes in Math., 920, Springer, 1982.

[9] S. Orey and W. Pruitt, Sample functions of the $N$-parameter Wiener process, Ann. Probability, 1 (1973), 138-163.

[10] D. W. Stroock, The Malliavin calculus and its applications, Stochastic integrals, Lecture Notes in Math., 851, Springer, 1981.

[11] M. Takeda, $(r, p)$-capacity on the Wiener space and properties of Brownian motion, to appear.

[12] G. Zimmerman, Some sample function properties of the two-parameter Gaussian process, Ann. Math. Statist, $43(1972)$, 1235-1246. 
Masatoshi FukUSHIMA

College of General Education Osaka University

Toyonaka, Osaka 560

Japan 